\title{
Simulation Training of E-Sports Players Based on Wireless Sensor Network
}

\author{
Feng Qian \\ Department of Physical Education, Suzhou University of Science and Technology, Suzhou, 215009 Jiangsu, China \\ Correspondence should be addressed to Feng Qian; qianfeng@mail.usts.edu.cn
}

Received 21 April 2021; Revised 22 June 2021; Accepted 28 July 2021; Published 18 August 2021

Academic Editor: Wenqing Wu

Copyright ( 2021 Feng Qian. This is an open access article distributed under the Creative Commons Attribution License, which permits unrestricted use, distribution, and reproduction in any medium, provided the original work is properly cited.

\begin{abstract}
The application of wireless sensors in sports competitions is becoming more and more common. This research mainly discusses the simulation training of e-sports players based on a wireless sensor network. Under the same experimental conditions, in order to avoid mutual influence and interference between the hand grip test and continuous endurance load, the exercise experiment for each subject was repeated twice. At the same time, the EMG signal collection and reaction time test during the endurance load are performed. All tests are data records before and after 40 minutes of the DOTA game competition. Before the start of each experimental test, the experimental equipment is calibrated and the parameters of the required indicators are set; the software was opened to run and checked whether it is normal; before the measurement, let the subjects perform simple preparation activities, train the subjects, and understand and be familiar with the action essentials required by the test to reduce the error. The original surface EMG signal recorded directly uses the built-in signal processing function in the MR-XP 1.08 master edition software to perform full-wave rectification and smoothing. Processing of original EMG data: firstly, the EMG signal during endurance contraction is intercepted. In order to exclude individual differences in sEMG indicators of different subjects, the starting point is the first rise of each subject to $60 \% \mathrm{MVC}$ or $25 \%$ MVC. The arrival time is the end point. In e-sports, the reaction speed when the prompt is effective is significantly faster than when the prompt is invalid $(p<0.05)$. At this time, the time interval between the cue prompt and the target stimulus is $500 \mathrm{~ms}$. This study is helpful to improve the athletes' technical and tactical level.
\end{abstract}

\section{Introduction}

The wireless sensor network is a highly application-related network. For different application requirements, the hardware structure of the wireless sensor network node is not the same, but its core components are basically the same. In certain environments, certain nodes require higher energy, stronger communication distance and processing capabilities, and even include GPS positioning functions.

A scientific training plan is an important part of highlevel training and a prerequisite for coaches to achieve their training goals. The introduction of information technology into the work of formulating scientific training plans can not only reduce the time spent on statistics by coaches and improve work efficiency but also quickly grasp and complete systematic and detailed information in time.

Shen et al. studied the reliability and applicability of active and continuous smart phone authentication using motion sensor behavior in various operating scenarios and systematically evaluated the uniqueness and durability of the behavior. Although they provide accurate and finegrained representations of user touch actions, their research process lacks data [1]. Yurtman and Barshan proposed a novel noniterative direction estimation method based on the physical and geometric characteristics of acceleration, 
angular velocity, and magnetic field vector to estimate the direction of the motion sensor unit. They obtain the orientation of the sensor unit according to the rotation quaternion transformation between the sensor unit frames. They evaluate the proposed method by incorporating it into the activity identification scheme for daily and sports activities. Although their scheme needs to accurately estimate the direction of the sensor unit to achieve the invariance of the direction the unit is worn on the body, the research process lacks experimental data. Signature recognition is to identify the owner of the signature, and verification is the process of finding the authenticity of the signature. Although both are important in the field of forensic science, verification is even more important for banks and credit card companies [2]. Behera et al. proposed a method to analyze 3D signatures captured using leap motion sensors. They extended the original 2D function from the original signature to 3D and applied a well-known classifier for identification and verification. Although they used the leap motion interface to create a large data set containing more than 2,000 signatures registered by 100 volunteers, their research method is still not new enough [3]. Motion sensor technology and automatic fall detection systems have become reliable and low-cost solutions for falls. $\mathrm{Yu}$ et al. has developed a fall detection system based on the hidden Markov model (HMM), which can use a single motion sensor to automatically detect falls for actual home monitoring scenarios. They proposed a new representation for the acceleration signal in HMM to avoid feature engineering. The HMM classifier is trained to detect falls based on the acceleration signal data collected from the motion sensor. Although they collect data sets from experiments that simulate falls and normal activities, the research process lacks theoretical foundation [4].

This research mainly discusses the simulation training of e-sports players based on a wireless sensor network. Under the same experimental conditions, in order to avoid mutual influence and interference between the hand grip test and continuous endurance load, the exercise experiment for each subject was repeated twice. The first test is for grip strength and finger flexibility, and the second test is done after two days of rest. At the same time, the EMG signal collection and reaction time test during endurance load are performed. All tests are data records before and after 40 minutes of the DOTA game competition. Before the start of each experimental test, the experimental equipment is calibrated and the parameters of the required indicators are set; the software was opened to run and checked whether it is normal; before the measurement, let the subjects perform simple preparation activities, train the subjects, and understand and be familiar with the action essentials required by the test to reduce the error. The original surface EMG signal recorded directly uses the built-in signal processing function in the MR-XP 1.08 Master Edition software to perform fullwave rectification, smoothing, calculation, and analysis of the original signal. The processing of the original EMG data is as follows: first, intercept the EMG signal during endurance contraction, in order to exclude the individual differences in sEMG indicators of different subjects, with each subject's first rise to $60 \%$ MVC or $25 \%$ MVC as the starting point; the arrival time is the end point. This research helps to improve the technical and tactical level of athletes.

\section{Simulation Training of E-Sports Players Based on Wireless Sensor Network}

\subsection{Related Technologies of Wireless Sensor Network}

2.1.1. Wireless Sensor Network. A wireless sensor network is a multihop self-organizing network system formed by deploying a large number of sensor nodes in a certain area through wireless network communication. Its purpose is to cooperate and sense, collect, process, and transmit the sensing objects in the network coverage area. [5-7]. The schematic diagram of the wireless sensor network structure is shown in Figure 1.

2.1.2. Target Tracking Technology. Target tracking can be divided into active active and passive passive according to the sensor type. In practical applications, passive passive multitarget tracking is more practical. Active active target tracking refers to obtaining the position information of the monitored target at any time through the cooperation of sensor nodes in the network when the target is actively carrying the transmitter. This method requires the cooperation of the tracked target to a certain extent. Active active target tracking is basically divided into two types based on ranging and nonranging. In practical applications, the monitored target generally does not actively carry active devices, and the main purpose of people deploying wireless sensor networks is to monitor some unknown things, so passive passive target tracking is more important [8]. Compared with singletarget tracking, multitarget tracking is more complicated, faces greater difficulties, and involves more technologies. The basic idea of multitarget tracking is to divide the entire tracking process into three stages. In order to eliminate ambiguity, it is necessary to introduce methods such as source separation and target classification. The third stage is singletarget tracking when the target is separated. At present, the research on multitarget tracking technology is a hot topic in the world. The main research content includes sensor node task allocation, data fusion, multitarget identification, and saving network resources. It is necessary to make breakthroughs in target detection and classification, state estimation, and key technologies such as forecasting and data association [9].

2.1.3. Target Activity Trajectory Mining Technology. The trajectory of the target refers to the path formed after the target has been active in the wireless sensor network for a period of time. Trajectory mining is a technology that obtains the true path by analyzing the sensor data triggered by the target within the time interval. At present, the target activity trajectory mining technology has become one of the research hotspots in the field of data mining and WSN target tracking. There are many methods for trajectory mining, which are mainly divided into three types: mining based on the overall trajectory, mining based on local trajectory similarity, and 


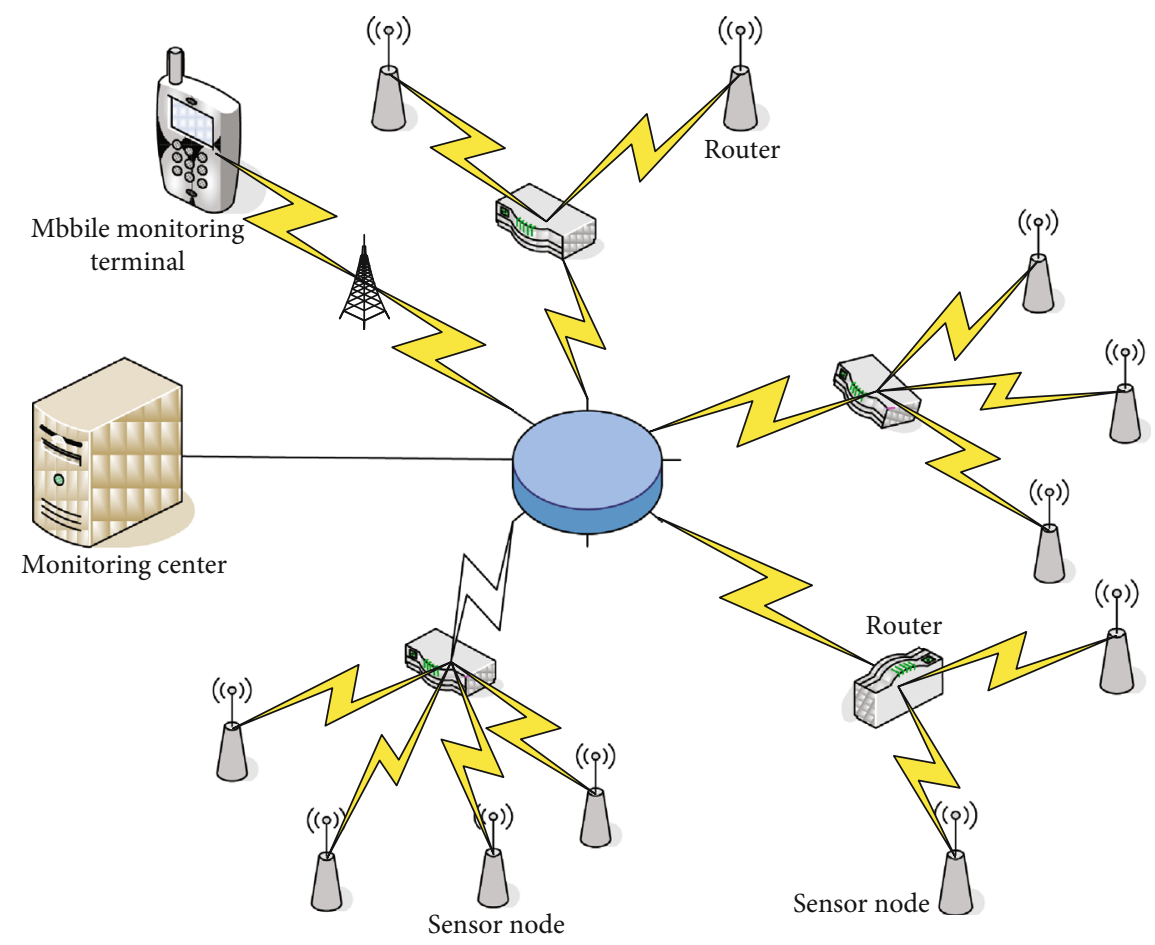

Figure 1: Schematic diagram of the wireless sensor network structure.

mining based on time and space information. The main goal is to realize the prediction and estimation and cluster analysis of target trajectories to form a reliable trajectory pattern library, while the trajectory mining method based on time and space information is more. To be flexible, it can not only well achieve the goals of the above two methods but also the mining method is flexible and suitable for a variety of applications.

\subsection{Wireless Sensor Network Algorithm}

2.2.1. LEACH Algorithm. In the initialization phase of a cycle, the system will randomly generate a random value from 0 to 1 and compare this value with the threshold $T(n)$. If the value is between $T(n)$ and 1, then the node is a normal node; otherwise, this node is the cluster head node [10]. $T(n)$ is calculated using the following method $[11,12]$ :

$$
T(n)=\left\{\begin{array}{l}
\frac{P}{1-P \times(r \bmod (1 / P))}, n \in G, \\
0, \text { if } n \notin G,
\end{array}\right.
$$

where $P$ represents the ratio of the cluster head node to all nodes in the network. The value of $P$ changes with different networks and is not fixed. $r$ represents the number of cycles currently in progress, and $G$ represents the set of nodes that have not been selected as cluster head nodes in the past $1 / P$ cycles. The generating formula of $T(n)$ ensures that every node in $1 / P$ cycles can become the cluster head node $[13,14]$.
2.2.2. The Cluster Head Selection of LEACH Algorithm. The work efficiency of wireless sensor networks is related to many factors, such as the internal energy consumption of network nodes, the distribution of nodes, and the distance between nodes. For a wireless sensor network, the LEACH algorithm actually has an optimal range for the number of clusters that should be divided. In the process of constructing a cluster, we should try to ensure that the number of cluster heads is within this optimal position. In this way, the working efficiency of the entire wireless sensor network is improved. The energy consumption of each node in the entire network was analyzed. The energy consumed by the cluster head includes data transmission, data fusion, and data reporting. We assume that the total energy consumption of the cluster head node in each cycle is [15]

$$
E_{\mathrm{CH}}=l E_{\mathrm{elec}} \frac{N}{k}+l E_{\mathrm{DA}} \frac{N}{k}+l \varepsilon d_{\mathrm{toCH}}^{4} .
$$

Assuming that in a cycle, the cluster head node and the ordinary nodes in the cluster communicate with each other, and the energy required to be consumed is expressed as $[16,17]$

$$
E_{\text {non-CH }}=l H_{\text {elec }}+l H_{\text {friss-amp }} d_{\text {toCH }}^{2} .
$$

Here, we believe that the entire wireless sensor network divides all nodes into $S$ clusters according to the node distribution area $k$, so the area occupied by each cluster can be expressed as $S / k$. At the same time, it is assumed that the density function of the node distribution within this cluster can mean $P(x, y)$; then, the distance 
between each node and the cluster head node is expressed as $d_{\mathrm{toCH}}$, and the mathematical expectation of $d_{\mathrm{toCH}}, d_{\mathrm{toCH}}$ can be expressed by the following formula [18]:

$$
E\left[d_{\mathrm{toCH}}^{2}\right]=\iint\left(x^{2}+y^{2}\right) \rho(x, y) d x d y=\iint r^{2} \rho(r, \theta) r d r d \theta
$$

Here, we regard the area of one of the clusters as a circular area with a radius of $R=S / \sqrt{\pi k}$. At the same time, the density distribution function is a constant of $\rho(r, \theta)$ with respect to $r$ and $\theta$. Then, the distance between each node and the cluster head node, $d_{\text {toCH }}$, can be expressed as $[19,20]$ :

$$
E\left[d_{\mathrm{toCH}}^{2}\right]=\rho \int_{\theta=0}^{2 \pi} \int_{r=0}^{S / \sqrt{\pi k}} d r d \theta=\frac{\rho}{2 \pi} \frac{S^{4}}{k^{2}} .
$$

Assuming that in the circular area, all sensor nodes are evenly distributed; according to the mathematical principle, there is $d_{\mathrm{toCH}}$; then, the mathematical expectation of the distance between a node and the cluster head node can be expressed as [21]

$$
E\left[d_{\mathrm{toCH}}^{2}\right]=\frac{1}{2 \pi} \frac{S^{2}}{k} .
$$

That is,

$$
E_{\text {non-CH }}=l H_{\text {elec }}+l H_{\text {friss-amp }} \frac{1}{2 \pi} \frac{S}{k} \text {. }
$$

Then, the energy consumed by ordinary nodes in the cluster can be expressed as $[22,23]$

$$
E_{\text {cluster }}=E_{\mathrm{CH}}+\frac{N}{k} E_{\text {non- } \mathrm{CH}} \text {. }
$$

Then, in the wireless sensor network, the total energy consumed by a single-cluster head node in a cycle is [24]

$$
E_{\text {round }}=k E_{\text {cluster }}=I\left(N E_{\text {elec }}+N E_{\mathrm{DA}}+k \varepsilon_{\text {two-ray-amp }} d_{\text {toBS }}^{4}+N \varepsilon_{\text {friss-amp }} \frac{1}{2 \pi} \frac{S^{2}}{k}\right),
$$

where $d_{\text {toCH }}$ represents the number of clusters in the wireless sensor network, and $d_{\text {toCH}}$ represents the average distance from the cluster head node to the network transmission base station. Take the derivative of $k$ in this formula and set the value of the partial derivative function to 0 . In a wireless sensor network, the optimal number of clusters should be $[25,26]$

$$
k=\sqrt{\frac{N}{2 \pi}} \sqrt{\frac{\varepsilon_{\text {feiss-amp }} S}{\varepsilon_{\text {two-ray-amp }} d_{\text {toBS }}^{2}}} .
$$

According to this formula, for a wireless sensor network within a specific range, we can more accurately calculate the number of optimal clusters that should be divided [27].
2.2.3. LEACH Algorithm Energy Consumption Model. When the distance between two nodes in the wireless sensor network is $d$, and they transmit data to each other, the length of the message transmitted is 1 bit; then, the energy consumed by the information node can be expressed as

$E_{\mathrm{tx}}(l, d)=E_{\mathrm{tx}-\mathrm{elec}}(l)+E_{\mathrm{tx}-\mathrm{amp}}(l, d)=\left\{\begin{array}{l}l E_{\mathrm{elec}}+l e_{f s} d^{2}, d<d_{0} \\ l E_{\mathrm{elec}}+l e_{f s} d^{2}, d<d_{0}\end{array}\right.$

The energy consumed by the receiving information node can be expressed as

$$
E_{\mathrm{tx}}(l)=E_{\mathrm{tx}-\mathrm{elec}}(l)=l E_{\text {elec }}
$$

In the above formula, $E_{\text {elec }}$ represents the energy consumed in the process of node sending unit data and node receiving unit data. In the above model, the two are equal in value. The monitoring area of each sensor node is $\pi R^{2}$, and the probability that each sensor can monitor the entire area is

$$
p=\frac{\pi R^{2}}{S}
$$

Then, the coverage probability of $n$ nodes is

$$
P(A+A+\cdots+A)=1-(1-p)^{n} .
$$

The number of randomly distributed sensor nodes is [28]

$$
n=\log _{\left(1-\pi R^{2}\right) / S}^{1-p}=\lg \frac{1-p}{\left(1-\pi R^{2}\right) / S} .
$$

\section{Experimental Design of Simulation Training for E-Sports Players}

3.1. Subjects. Select 15 boys in the professional e-sports class of a college, aged between 20 and 24 years old (average age: $22.7 \pm 2.8$ years old), height: $174.6 \pm 3.2 \mathrm{~cm}$, weight: $65.7 \pm$ $9.6 \mathrm{~kg}$, and engaged in DOTA special training 1-4 years (average age: $2.2 \pm 1.3$ years). He was in good physical health, had no strenuous exercise 24 hours before the experiment, had not used a computer for a long time, in good mental state, not lacking sleep, and no malaise and other bad states. The subjects used the right hand to operate the mouse during the game. Before the experiment, the subjects were briefly explained with the subjects of the experiment procedures, experimental requirements, etc., so that the subjects understood the basic procedures of the experiment and signed the informed consent form voluntarily.

3.2. Experimental Instruments and Methods. Under the same experimental conditions, in order to avoid mutual influence and interference between the hand grip test and continuous endurance load, the exercise experiment for each subject was repeated twice. The first test is for grip strength and 
finger flexibility, and the second test is done after two days of rest. At the same time, the EMG signal collection and reaction time test during endurance load are performed. All tests are data records before and after 40 minutes of the DOTA game competition. Before the start of each experimental test, the experimental equipment is calibrated and the parameters of the required indicators are set; the software to run was opened and checked whether it is normal; before the measurement, let the subjects perform simple preparation activities, train the subjects, and understand and be familiar with the action essentials required by the test to reduce the error.

3.2.1. Grip Strength Test. Before the test, adjust the grip distance according to the size of the testee's hand and bend the second joint of the middle finger by 90 degrees to fully exert force. During the test, the subject is required to stand upright, with the two feet naturally separated by about one foot distance, the arms are naturally drooped, the palms are inward, the display window of the grip meter is facing outwards, and then the inner and outer grips are fully held. The instrument displays the maximum grip strength and maintains it. Do not touch the dynamometer with your body or clothing.

3.2.2. Finger Flexibility Test. In the experiment, considering that the familiarity of each subject with tweezers is different, and it takes too long to insert a complete hole in the experiment plate and it takes too long to test 3 times in a row, the subject's patience will affect the accuracy of the data, in order to avoid these situations The error caused by this experiment requires the subject to directly insert the metal rod from the first hole in the upper left corner by hand. The start and end of the experiment were manually pressed by the tester.

3.2.3. Response Time Test. First, press the simple response button (the experiment will present a stimulus), instruct the subject to look at the stimulus light source light, and pay attention to waiting for the signal to be sent. The preparation signal light below the light turns on first. When the stimulus light is on, press the red button at the fastest speed. The timing of the display window is stopped, waiting for the next signal to be sent; there are 5 signals in total. Each time a stimulus is presented, the number of responses is displayed during the test, and when the response is wrong, the number of errors is displayed. At the end of the experiment, the average response time and the number of errors are displayed.

3.2.4. Test Method of EMG Signal and Acquisition of Original EMG Signal. The surface EMG collection using the surface EMG telemetry system is shown in Figure 2. The sampling frequency is $1000 \mathrm{~Hz}$, the preamplifier gain value is 1000 , the input impedance $>100 \mathrm{Mohm}$, the common mode rejection ratio $>100 \mathrm{~dB}$, the channel sampling bandwidth is 10 $500 \mathrm{~Hz}$, and the sensitivity is $1 \mathrm{~V}$.

3.3. Processing and Analysis of Surface EMG. The original surface EMG signal recorded directly uses the built-in signal processing function in the MR-XP 1.08 Master Edition software to perform full-wave rectification, smoothing, calculation, and analysis of the original signal. The processing of

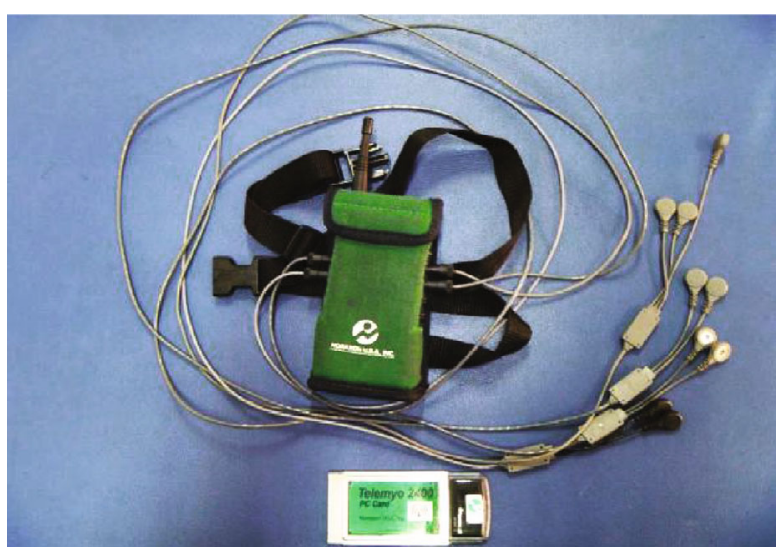

FIgURE 2: Surface EMG telemetry system.

TABLE 1: The relationship between packet loss rate and communication distance.

\begin{tabular}{lccc}
\hline $\begin{array}{l}\text { Communication } \\
\text { distance }(\mathrm{m})\end{array}$ & $\begin{array}{c}\text { Number of } \\
\text { packets sent }\end{array}$ & $\begin{array}{c}\text { Number of } \\
\text { received packets }\end{array}$ & $\begin{array}{c}\text { Packet loss } \\
\text { rate }(\%)\end{array}$ \\
\hline 10 & 53645 & 53450 & $0.36 \%$ \\
15 & 62850 & 62511 & $0.54 \%$ \\
20 & 63224 & 62635 & $0.93 \%$ \\
25 & 55628 & 49275 & $11.42 \%$ \\
30 & 58605 & 44978 & $23.25 \%$ \\
35 & 63542 & 30665 & $51.74 \%$ \\
40 & 65826 & 1527 & $97.68 \%$ \\
\hline
\end{tabular}

the original EMG data is as follows: first, intercept the EMG signal during endurance contraction, in order to exclude the individual differences in sEMG indicators of different subjects, with each subject's first rise to $60 \%$ MVC or $25 \% \mathrm{MVC}$ as the starting point; the arrival time is the end point.

3.4. Statistical Processing. Statistical analysis was performed with SPSS 13.0 statistical software. The significance test of the difference was performed by one-way analysis of variance, the difference between the two groups was tested by LSD-t, and the statistics of simulation training results of esports players were performed by a group $t$-test. $p<0.05$ is considered to be significant and statistically significant.

\section{Results and Discussion}

Select the serial port baud rate of $57600 \mathrm{bps}$, after writing the test data to the node, send it through the CC2420 radiofrequency, and send the data received by the receiving end to the PC serial port, and receive the data through the serial port tool. The data sent, received data, and communication distance are measured in the experiment. The relationship is shown in Table 1 . When the communication distance between nodes is about $20 \mathrm{~m}$, the communication can be roughly regarded as reliable. When the node communication distance increases to more than $30 \mathrm{~m}$, the node packet loss rate increases rapidly. When it approaches $40 \mathrm{~m}$, the network is almost interrupted. 


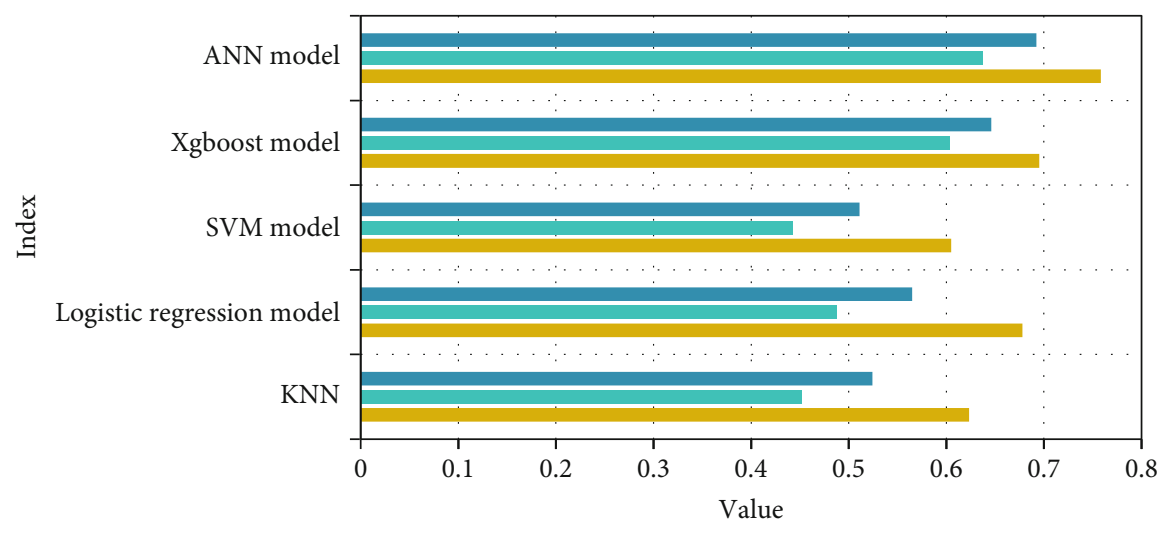

F1 value

Recall rate $\mathrm{R}$

Accuracy P

Figure 3: Comparison of performance indicators of each model.

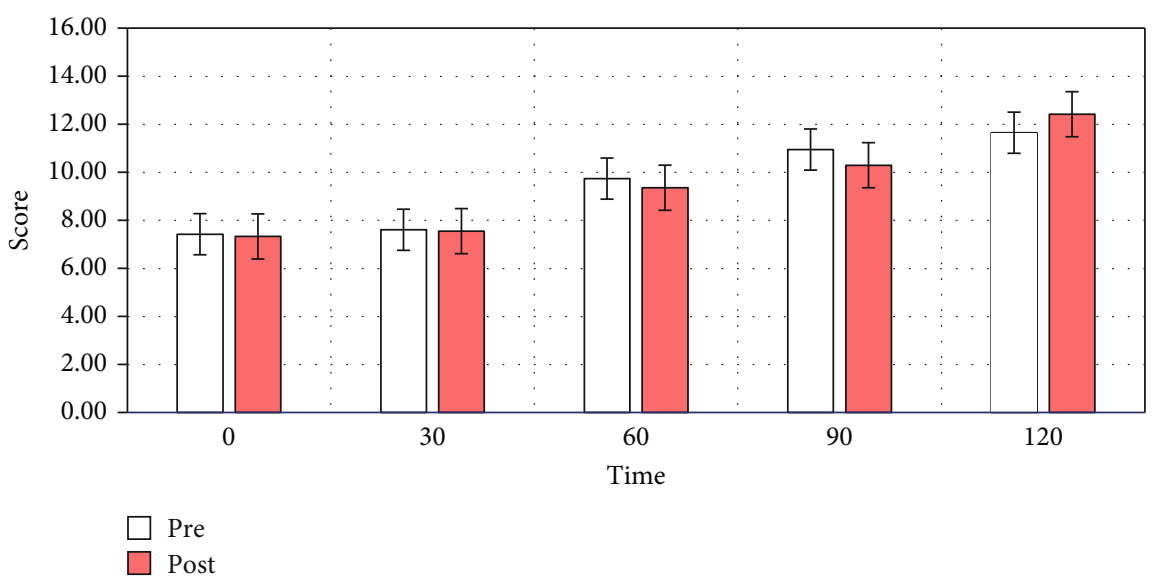

FIgURE 4: Changes in endurance load time and subjective fatigue feeling of flexor superficial muscle (FDS).

The performance index comparison of each model is shown in Figure 3. Using traditional one-hot encoding (one-hot) input data, combined with traditional machine learning algorithms (excluding neural network models), the integrated tree regression algorithm represented by XGBoost performs the most eye-catching, not only the accuracy rate is as high as 0.788 but also the calculation is the fastest speed, only 1.67 hours. In contrast to other highly explanatory algorithms, the logistic regression algorithm has the highest accuracy rate of only 0.690, and it takes 4.17 hours. The KNN algorithm is not ideal because of its simplicity. It can be seen from the figure that the related time consumption is a concern. Due to the parallel and multithreading of the algorithm itself, the speed of XGBoost is the fastest among all models, and it processes about $80 \mathrm{~W}$ of data in about 1.5 hours.

Figure 4 shows the changes in the endurance load time and subjective fatigue of the finger superficial flexor muscles (FDS). With the extension of the mouse click duration, the subject's subjective fatigue score gradually increased. When the endurance contracted for 30 seconds after exercise, there was no significant change; when contracted to $60 \mathrm{~s}$, compared with before exercise, the subjective fatigue feeling was significantly increased compared with before exercise $(p<0.05)$. At 90s, the subjective fatigue feeling was higher than before exercise. The difference is significant $(p<0.01)$, and at $120 \mathrm{~s}$, there is a significant increase compared to before exercise $(p<0.05)$.

The selection of e-sports athletes is also different from the selection of traditional competitive sports. E-sports players often do not need excellent physical conditions, and they pay more attention to the eye-hand coordination ability, reaction speed, and thinking ability of the players during the selection. E-sports is a new type of sports under the conditions of the times and technological development, and it has a broader development prospect and future. The amplitude values of the surface EMG signal of the extensor muscle at each time point of $25 \%$ MVC endurance contraction are shown in Table 2. The amplitude value of the surface EMG signal before exercise gradually increases with time; $30 \mathrm{~s}$ after exercise is slightly lower than the amplitude value at the beginning of exercise, and after $60 \mathrm{~s}$, the amplitude value 
TABLE 2: 25\% MVC endurance contraction at each time point of the amplitude of the surface EMG signal of the extensor muscle.

\begin{tabular}{lccccc}
\hline & 0 & $30 s$ & $60 s$ & $90 s$ & $120 s$ \\
\hline Before exercise & $48.52 \pm 16.0$ & $65.55 \pm 19.12$ & $72.12 \pm 26.20$ & $74.95 \pm 25.74$ & $83.77 \pm 36.07$ \\
After exercise & $65.68 \pm 34.4$ & $64.62 \pm 20.74$ & $72.73 \pm 22.67$ & $94.91 \pm 51.55$ & $109.7 \pm 56.6$ \\
\hline
\end{tabular}

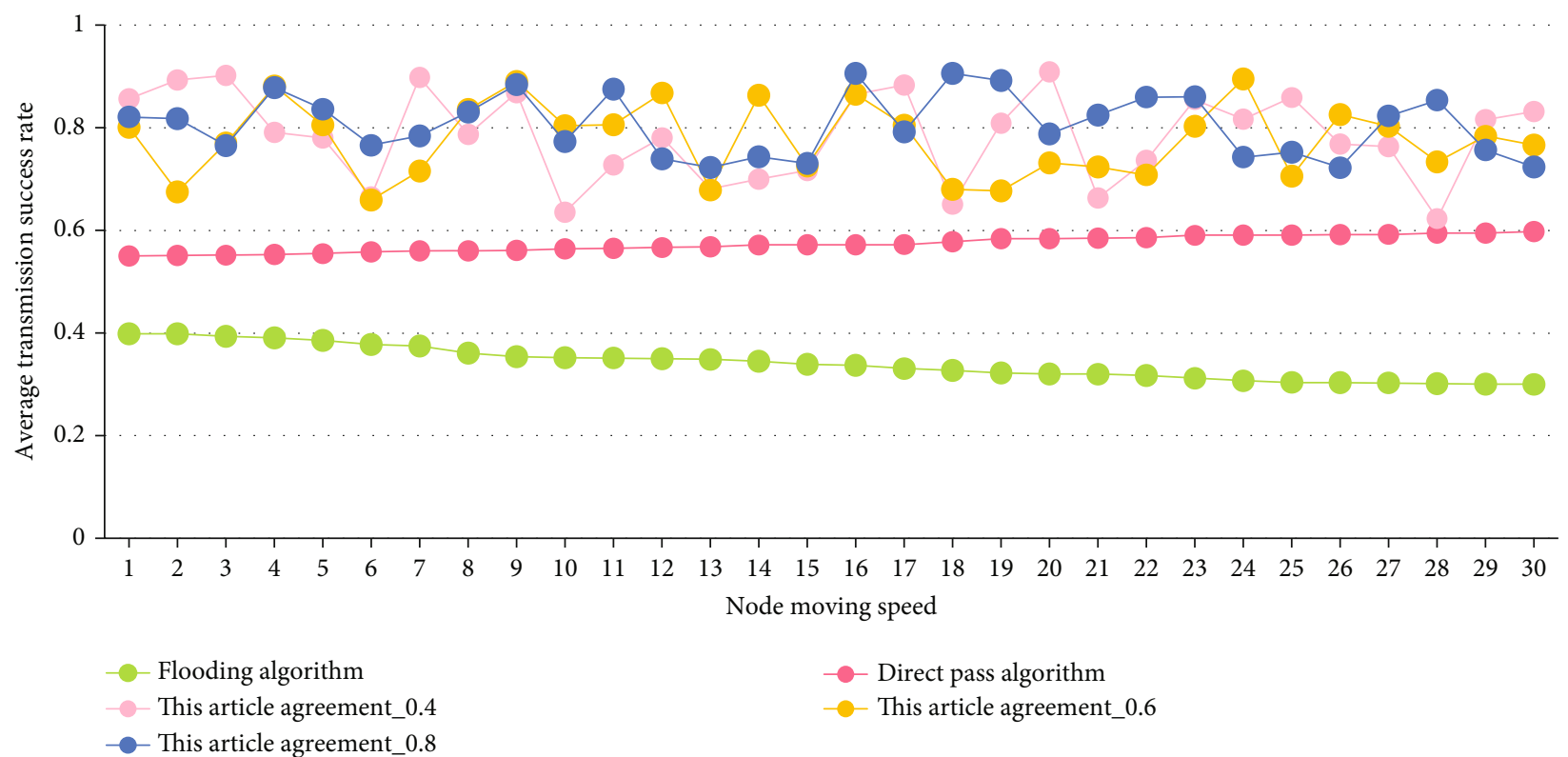

Figure 5: The effect of node moving speed on the average transmission success rate.

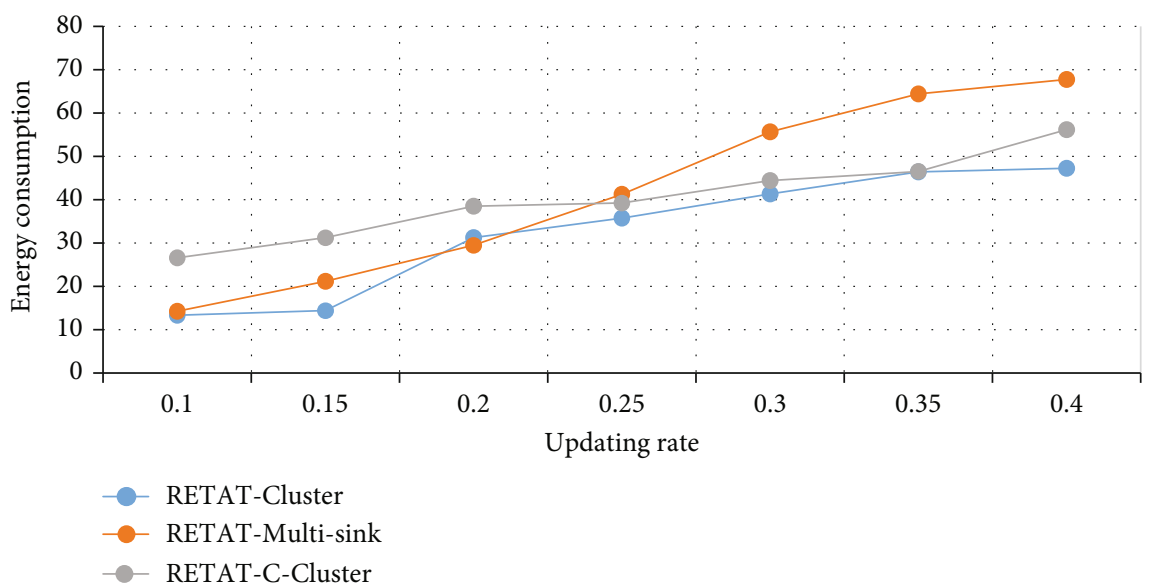

FIGURE 6: Energy consumption of three transmission task allocation strategies under different network update ratios.

gradually increases with the continuation of time, and at $120 \mathrm{~s}$ and before exercise, compared with a significant increase $(p<0.05)$.

The influence of node moving speed on the average transmission success rate is shown in Figure 5. With the increase of the node's moving speed, the average information transmission success rate of the protocol and the direct delivery algorithm in this paper has been continuously improved, while the average information transmission success rate of the flooding algorithm has gradually decreased. This is because in the protocol and direct deliv- ery algorithm in this article, as the speed of the node increases, the communication opportunities between nodes and between nodes and base stations are increased, and the information needs to be forwarded. The node has more opportunities to choose a suitable relay node. To forward the message, however, in the flooding algorithm, the nodes move too fast, which leads to the increase of communication conflicts and reduces the performance of the network. In addition, we can see from the figure that the initial importance still affects the average success rate of information transmission. 


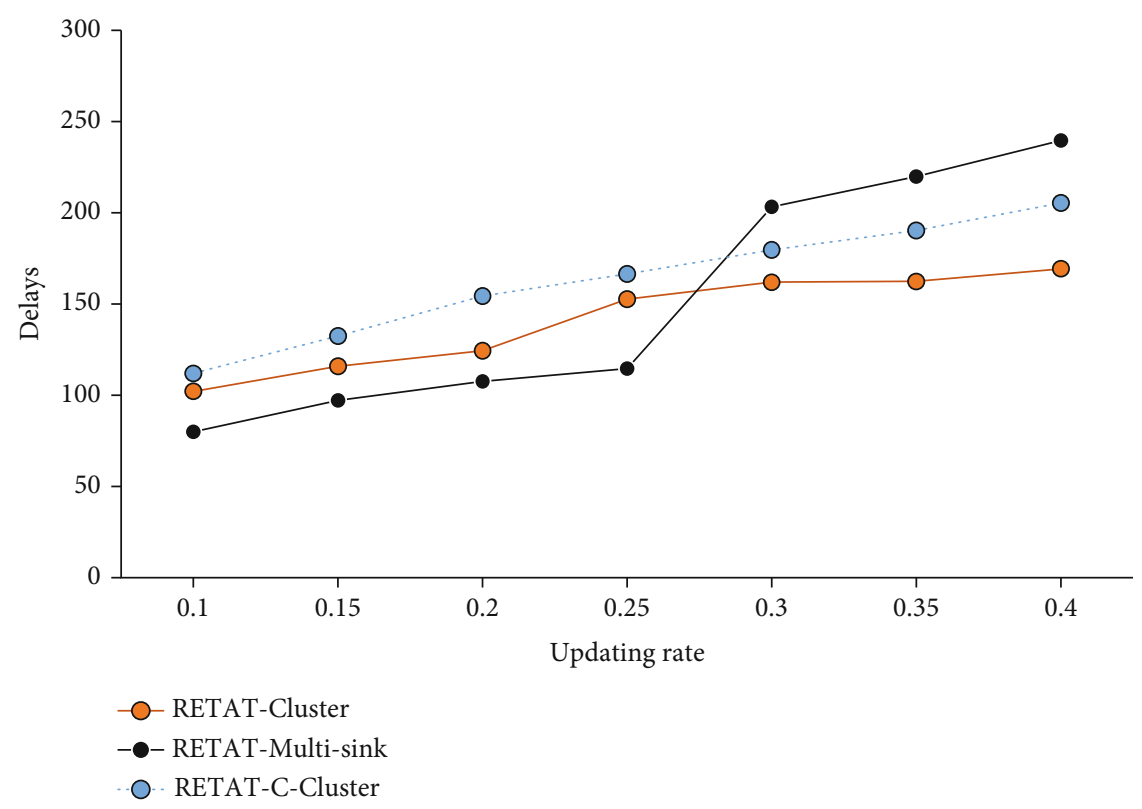

FIGURE 7: Delay of transmission task allocation under different network update frequencies.

TABLE 3: Analysis of the difference in response time of clue prompts.

\begin{tabular}{|c|c|c|c|c|c|c|}
\hline Sample book & Effective reminder & Invalid prompt & Difference & $t$ & $d f$ & $p$ \\
\hline ESports player & $580.53 \pm 70.70$ & $615.02 \pm 98.70$ & -34.98 & -2.568 & 29 & 0.015 \\
\hline Traditional athletes & $672.02 \pm 79.96$ & $725.28 \pm 110.64$ & -53.26 & -3.453 & 29 & 0.002 \\
\hline Ordinary college students & $654.86 \pm 100.78$ & $717.38 \pm 97.78$ & -62.52 & -3.329 & 29 & 0.002 \\
\hline
\end{tabular}

The energy consumption of the three transmission task allocation strategies under different network update ratios is shown in Figure 6. It can be seen that as the network update rate increases, the energy consumption of the three transmission task distribution increases significantly as the network update rate increases. Since the network update in RETAT$\mathrm{C}$-cluster has to be aggregated to the only sink after long distances and multiple hops, which increases the communication load of the network, in most cases, the energy consumption of the RETAT-C-cluster of the three strategies is directly higher than the other two distributed transmission task allocation methods. As the network update rate is greater than $25 \%$, the energy consumption of RETATmultisink increases rapidly, surpassing RETAT-C-cluster and RETAT-cluster to become the highest energy consumption of the three strategies. Because of the high network update rate, the exchange of global views between multiple sinks in RETAT-multisink and the operation of ensuring the consistency of the global view all lead to a rapid increase in energy consumption.

The delay of transmission task allocation under different network update frequencies is shown in Figure 7. With the increase of the network update rate, the delays of the three kinds of transmission tasks are all on the rise. In the current network simulation parameter environment, statistics found that when the network update rate is lower than $25 \%$, the delays of both RETAT-cluster and RETAT-multisink are less than RETAT-C-cluster. This is because when there are a small amount of updates in the network, the first two are processed locally without affecting the reliability of the mission target, and the entire network is not required to be updated, so the delay is low. When the network update rate exceeds $25 \%$, the delay of RETAT-multisink increases rapidly and is higher than that of RETAT-C-cluster, which is caused by the operation to ensure the consistency of the global view under the high update rate.

E-sports is an adversarial competitive sports event, which requires players from both sides to arrive at the designated venue and compete in skill and physical stamina under fair and just referee supervision and competition rules. However, traditional online games are only used for game entertainment on dedicated servers provided by network operators, without the performance of the spirit of sports competition. When there are 3 distracting stimuli, the response time is the dependent variable, and the effective prompt and invalid prompt are the independent variables. Table 3 shows the results of the matched sample 1 test of e-sports athletes, traditional athletic athletes, and ordinary college students. In the three groups of participants, the response speed when the prompt is effective is significantly faster than when the prompt is invalid $(p<0.05)$. At this time, the time interval between the prompt and the target stimulus is $500 \mathrm{~ms}$, which shows that in the three groups of participants the facilitating effect of attention appeared in the time interval of $500 \mathrm{~ms}$. 


\section{Conclusion}

This research mainly discusses the simulation training of esports players based on a wireless sensor network. Under the same experimental conditions, in order to avoid mutual influence and interference between the hand grip test and continuous endurance load, the exercise experiment for each subject was repeated twice. The EMG signal collection and reaction time test during endurance load are performed. All tests are data records before and after 40 minutes of the DOTA game competition. Before the start of each experimental test, the experimental equipment is calibrated and the parameters of the required indicators are set; the software to run was opened and check whether it is normal; before the measurement, let the subjects perform simple preparation activities, train the subjects, and understand and be familiar with the action essentials required by the test to reduce the error. The original surface EMG signal recorded directly uses the built-in signal processing function in the MR-XP 1.08 Master Edition software to perform full-wave rectification, smoothing, calculation, and analysis of the original signal. The processing of the original EMG data is as follows: first, intercept the EMG signal during endurance contraction, in order to exclude the individual differences in sEMG indicators of different subjects, with each subject's first rise to $60 \%$ MVC or $25 \%$ MVC as the starting point. This research helps to improve the technical and tactical level of athletes.

\section{Data Availability}

No data were used to support this study.

\section{Conflicts of Interest}

There are no potential competing interests in our paper.

\section{Authors' Contributions}

The author has seen the manuscript and approved to submit to your journal.

\section{Acknowledgments}

This work was supported by the Philosophy and Social Science Research Project of Universities in Jiangsu Province (No. 2020SJA1391).

\section{References}

[1] C. Shen, Y. Li, Y. Chen, X. Guan, and R. A. Maxion, "Performance analysis of multi-motion sensor behavior for active smartphone authentication," IEEE Transactions on Information Forensics \& Security, vol. 13, no. 1, pp. 48-62, 2018.

[2] A. Yurtman and B. Barshan, "Novel noniterative orientation estimation for wearable motion sensor units acquiring accelerometer, gyroscope, and magnetometer measurements," IEEE Transactions on Instrumentation and Measurement, vol. 69, no. 6, pp. 3206-3215, 2020.
[3] S. K. Behera, D. P. Dogra, and P. P. Roy, "Analysis of 3D signatures recorded using leap motion sensor," Multimedia Tools and Applications, vol. 77, no. 11, pp. 14029-14054, 2018.

[4] S. Yu, H. Chen, and R. A. Brown, "Hidden Markov modelbased fall detection with motion sensor orientation calibration: a case for real-life home monitoring," IEEE Journal of Biomedical and Health Informatics, vol. 22, no. 6, pp. 1847-1853, 2018.

[5] M. Hirata, R. Watanabe, Y. Koyano et al., "Using a motion sensor-equipped smartphone to facilitate CT-guided puncture," Cardiovascular \& Interventional Radiology, vol. 40, no. 4, 2017.

[6] J. K. Min, E. J. Cheon, J. M. Kim, S. C. Lee, and K. Choi, "Comparison of the 6-DOF motion sensor and stain gauge data for ice load estimation on IBRV ARAON," Journal of the Society of Naval Architects of Korea, vol. 53, no. 6, pp. 529-535, 2016.

[7] I. Butun, P. Österberg, and H. Song, "Security of the internet of things: vulnerabilities, attacks, and countermeasures," IEEE Communications Surveys \& Tutorials, vol. 22, no. 1, pp. 616644, 2020.

[8] M. Elhoseny, "Multi-object detection and tracking (MODT) machine learning model for real-time video surveillance systems," Circuits, Systems, and Signal Processing, vol. 39, pp. 611-630, 2020.

[9] I. I. Duma and S. Giurgiu, "Circadian activity and nest use of Dryomys nitedula as revealed by infrared motion sensor cameras," Folia Zoologica, vol. 61, no. 1, pp. 49-53, 2012.

[10] Z. Hong, R. Wang, and X. Li, “A clustering-tree topology control based on the energy forecast for heterogeneous wireless sensor networks," IEEE/CAA Journal of Automatica Sinica, vol. 3, no. 1, pp. 70-79, 2016.

[11] L.-B. Huang, W. Xu, C. Zhao et al., "Multifunctional water drop energy harvesting and human motion sensor based on flexible dual-mode nanogenerator incorporated with polymer nanotubes," ACS Applied Materials And Interfaces, vol. 12, no. 21, pp. 24030-24038, 2020.

[12] D. Ebrahimi and C. Assi, "On the interaction between scheduling and compressive data gathering in wireless sensor networks," IEEE Transactions on Wireless Communications, vol. 15, no. 4, pp. 2845-2858, 2016.

[13] J. M. Pak, C. K. Ahn, P. Shi, Y. S. Shmaliy, and M. T. Lim, "Distributed hybrid particle/FIR filtering for mitigating NLOS effects in TOA-based localization using wireless sensor networks," IEEE Transactions on Industrial Electronics, vol. 64, no. 6, pp. 5182-5191, 2017.

[14] F. Deniz, H. Bagci, I. Korpeoglu, and A. Yazıcı, “An adaptive, energy-aware and distributed fault-tolerant topology-control algorithm for heterogeneous wireless sensor networks," $\mathrm{Ad}$ Hoc Networks, vol. 44, no. 7, pp. 104-117, 2016.

[15] W. Liu, X. Zhou, S. Durrani, H. Mehrpouyan, and S. D. Blostein, "Energy harvesting wireless sensor networks: delay analysis considering energy costs of sensing and transmission," IEEE Transactions on Wireless Communications, vol. 15, no. 7, pp. 4635-4650, 2016.

[16] T. A. Donaldson, "Inline calibration of motion sensor," Journal of Chinese Pharmaceutical Sciences, vol. 48, no. 23, pp. 2022-2025, 2016.

[17] G. Marin, F. Dominio, and P. Zanuttigh, "Hand gesture recognition with jointly calibrated leap motion and depth sensor," Multimedia Tools and Applications, vol. 75, no. 22, pp. 14991-15015, 2016. 
[18] H. Jin, Q. Chen, Z. Chen, Y. Hu, and J. Zhang, "Multi-LeapMotion sensor based demonstration for robotic refine tabletop object manipulation task," Caai Transactions on Intelligence Technology, vol. 1, no. 1, pp. 104-113, 2016.

[19] Z. Wei, W. Zhang, and W. Liu, "Attitude theory and experimental research of micro-aircraft based on MEMS sensor," Piezoelectrics \& Acoustooptics, vol. 40, no. 4, pp. 516-520, 2018.

[20] D. Laurijssen, S. Truijen, W. Saeys, W. Daems, and J. Steckel, "An ultrasonic six degrees-of-freedom pose estimation sensor," IEEE Sensors Journal, vol. 17, no. 1, pp. 151-159, 2016.

[21] K. F. Chiang and H. H. Wang, "Nurses' experiences of using a smart mobile device application to assist home care for patients with chronic disease: a qualitative study," Journal of Clinical Nursing, vol. 25, no. 13-14, pp. 2008-2017, 2016.

[22] D. Korpi, J. Tamminen, M. Turunen et al., "Full-duplex mobile device - pushing the limits," IEEE Communications Magazine, vol. 54, no. 9, pp. 80-87, 2016.

[23] L. Zhou, "Mobile device-to-device video distribution," $A C M$ Transactions on Multimedia Computing Communications and Applications, vol. 12, no. 3, pp. 1-23, 2016.

[24] E. Noei, M. D. Syer, Y. Zou, A. E. Hassan, and I. Keivanloo, “A study of the relation of mobile device attributes with the userperceived quality of android apps," Empirical Software Engineering, vol. 22, no. 6, pp. 3088-3116, 2017.

[25] Y. Zou and G. Wang, "Intercept behavior analysis of industrial wireless sensor networks in the presence of eavesdropping attack," IEEE Transactions on Industrial Informatics, vol. 12, no. 2, pp. 780-787, 2016.

[26] J. Jiang et al., "Geographic multipath routing based on geospatial division in duty-cycled underwater wireless sensor networks," Journal of Network \& Computer Applications, vol. 59, no. 1, pp. 4-13, 2016.

[27] Z. Xu, L. Chen, C. Chen, and X. Guan, "Joint clustering and routing design for reliable and efficient data collection in large-scale wireless sensor networks," IEEE Internet of Things Journal, vol. 3, no. 4, pp. 520-532, 2016.

[28] S. Kumari, X. Li, F. Wu, A. K. Das, H. Arshad, and M. K. Khan, "A user friendly mutual authentication and key agreement scheme for wireless sensor networks using chaotic maps," Future Generation Computer Systems, vol. 63, no. 10, pp. 5675, 2016. 